\title{
Optimal surveillance against bioinvasions: The sample average approximation method applied to an agent-based spread model
}

\author{
Hoa-Thi-Minh Nguyen ${ }^{1}$, Pham Ha ${ }^{1}$, and Tom Kompas ${ }^{2}$ \\ ${ }^{1}$ Australian National University \\ ${ }^{2}$ University of Melbourne Australian Centre of Excellence for Biosecurity Risk Analysis
}

April 28, 2020

\begin{abstract}
Trade-offs exist between the point of early detection and the future cost of controlling any invasive species. Finding optimal levels of early detection, with post-border active surveillance, where time, space and randomness are explicitly considered, is computationally challenging. We use a stochastic programming model to find the optimal level of surveillance and predict damages, easing the computational challenge by combining the Sample Average Approximation approach and parallel processing techniques. The model is applied to the case of Asian Papaya Fruit Fly (PFF), a highly destructive pest, in Queensland, Australia. To capture the non-linearity in PFF spread, we use an agent-based model, which is calibrated to a highly detailed landuse raster map $(50 \mathrm{~m} \times 50 \mathrm{~m})$ and weather-related data, validated against a historical outbreak. We find that current surveillance levels are less than optimal.
\end{abstract}

\section{Hosted file}

Manuscript.pdf available at https ://authorea.com/users/309824/articles/440688-optimal-surveillanceagainst-bioinvasions-the-sample-average-approximation-method-applied-to-an-agent-based-spreadmodel 